\title{
PubMed Central as a platform for the survival of open-access biomedical society journals published in Korea
}

\author{
Sun Huh \\ Department of Parasitology and Institute of Medical Education, College of Medicine, Hallym University, Chuncheon, Korea
}

\begin{abstract}
Twenty-one years have passed since PubMed Central (PMC) launched. The present case study describes Korean editors' history of participation in PMC and their contributions to PMC. The three main turning points in the history of Korean editors' involvement with PMC were as follows: first, the production of PMC XML files and deposition starting in 2008; second, thorough evaluations of applying journals since 2014; and third, the feasibility of non-English journals being indexed in PMC starting in 2019. The importance of PMC is further shown by the fact that KoreaMed Synapse, a full-text XML database of biomedical journals in Korea that was launched in 2007, was created by benchmarking PMC. Scholarly societies or institutes publish 724 (34.2\%) of the 2,119 PMC journals without embargo in June 2021. Out of those 724 journals, 127 (17.5\%) are published in Korea. PMC has helped local journals receive more citations from researchers worldwide, increasing their likelihood of being indexed in international databases. The number of submissions from international researchers has increased, thereby making it possible for journals to achieve international diversity. As the best full-text platform of biomedical journals, PMC has provided an excellent opportunity for biomedical journal editors in Korea to change their journals' language to English and produce full-text JATS (Journal Article Tag Suite) XML files. These factors have made Korea the second-ranked country in terms of no-embargo PMC journals published by academic societies or institutes.
\end{abstract}

Keywords

Benchmarking; Language; Publications; PubMed Central; Republic of Korea

Received: June 6, 2021

Accepted: August 3, 2021

Correspondence to Sun Huh shuh@hallym.ac.kr

ORCID

Sun Huh

https://orcid.org/0000-0002-8559-8640

\section{Introduction}

Background/rationale: PubMed Central (PMC) is the full-text literature database of biomedical journals maintained by the National Center for Biotechnology Information of the US National Library of Medicine (NLM). It was established in February 2000, and 21 years have 
passed since the launch of the database. At first, two journals were listed: Proceedings of the National Academy of Sciences and Molecular Biology of the Cell. The number of PMC journals has subsequently increased year by year. The turning point of the acceleration of the number of journals was the deposition of open-access journals published by BioMedCentral, PLoS (Public Library of Science), Bentham Science Publishers, Hindawi Publishing Company, Frontiers Research Foundation, MedKnow Publication Company, and MDPI (Multidisciplinary Digital Publishing Institute). On May 26, 2021 , it was announced that "7,000,000 articles are archived in PMC. The content was provided by: 2,477 full participation journals, $332 \mathrm{NIH}$ portfolio journals, and 7,907 selective deposit journals" [1].

It is known that citations of local medical journals-especially from Korea-increase if they are included in PMC [2]. PMC is believed to be the best platform for local biomedical journals published in Korea to increase their citations. If a journal is deposited to PMC, the abstract is transferred to PubMed, an essential biomedical database for researchers throughout the world. Before the launch of PMC, to be indexed in PubMed, it was necessary for a journal to pass the MEDLINE evaluation. However, it is challenging for local journals to pass the review by the Literature Selection Technical Review Committee. In the year 2007, a number of MEDLINE journals from Korea was 14. In May 2021 only 33 journals from Korea were listed in MEDLINE. Therefore, most biomedical journal editors in Korea tried to add their journals to PMC as part of journal development initiatives. As of June 2021, the number of PMC journals without embargo from Korea has increased to 127 . It is, therefore, time to reflect on the history of the deposition of PMC XML files of biomedical journals from Korea and the contributions of Korean editors to PMC. The review of this history presented herein will provide valuable information for biomedical editors in Korea to continue to promote their journals to the international level.

Objectives: This study aimed to explain how biomedical society journal editors in Korea have used PMC as a platform for the survival of their journals and to demonstrate the extent to which their journals have contributed to PMC. Specifically, the following topics were addressed: first, major turning points in the addition of Korean biomedical journals to PMC; second, KoreaMed Synapse as a benchmarking of PMC; and third, the proportion of journals from Korea in PMC.

\section{Methods}

Ethics statement: This study is based on a literature database; therefore, neither institutional review board approval nor obtaining informed consent was required.
Study design: The present study consists of a historical review and descriptive study of PMC journals according to country.

Setting/Data sources/Measurement: Historical information was gathered from the author's experiences of PMC XML production and dissemination to other editors in Korea. The number of PMC journals and a more precise analysis according to the embargo period and country was done using the PMC journal list on June 1, 2021.

Statistical methods: Descriptive statistics were applied to the analysis of PMC journals according to the embargo period, publisher type, and country.

\section{Results}

Three turning points in the addition of Korean biomedical journals to PMC

First, production of PMC XML files and deposition: In 2005, I became the editor-in-chief of the Journal of Educational Evaluation for Health Professions. After publishing the journal for a year, I decided to change the journal's language to Englishonly in 2006 [3]. This decision was inevitable because the journal had only published nine articles in 2005. To maintain the publication of an appropriate number of articles, it was necessary for the journal to be able to receive manuscripts from all over the world. To disseminate the journal more widely, I also decided to add the journal to PMC. However, I had no information on PMC XML production companies at that time, and I was faced with the need to produce full-text PMC XML files by myself. In July 2006, I succeeded in producing PMC XML files for one article, which passed the validation test. The file was transformed into an HTML file through Xalan-C, an XSLT processor for transforming XML documents into HTML, text, or other XML document types. Because I served as the chair of the committee of information management of the Korean Association of Medical Journal Editors (KAMJE, https://kamje.or.kr), I began to train the staff and students taking part in internship in PMC XML production. On August 11, 2006, a PMC XML and XSLT Processor workshop was held at Sookmyung Women's University Library (Suppl. 1). This was the first step towards producing PMC XML in Korea. After the workshop, many staff and students were able to produce PMC XML files.

Many journal editors also started to change their journals' language to English-only to deposit their journals to PMC, reflecting the need to receive more citations [2]. After another workshop in July 2007 (Suppl. 2), PMC XML and Crossref XML were produced simultaneously. In August 2007, the Journal of the Korean Ophthalmological Society was the first society journal from Korea to achieve successful DOI and Crossref XML deposition. The Journal of Korean Medical Sci- 
ence became the first society journal from Korea to be deposited in PMC in November 2008. PMC accepted the Journal of Educational Evaluation for Health Professions in February 2009. As of June 10, 2010, a total of 29 journals from Korea were deposited in PMC. Korea was the fourth-ranking coun-

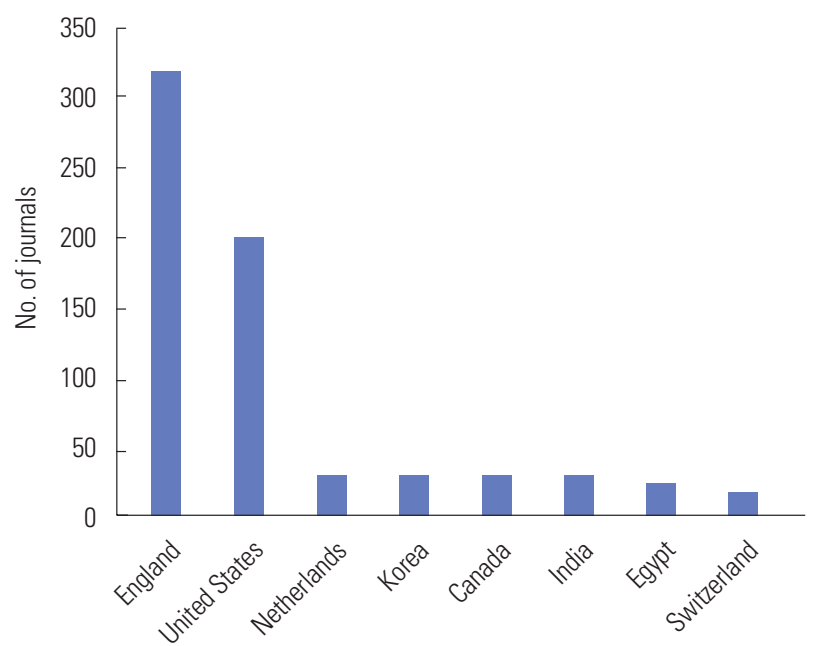

Fig. 1. The number of PubMed Central journals according to the place of publication on June 10, 2010. Reproduced from Huh S. J Korean Med Assoc 2010;53:659-67 under a Creative Commons Non-Commercial license (CC-BYNC) [4]. try in PMC at that time (Fig. 1) [4].

Second, through evaluation of the applying journals: In 2015, I started to receive emails from English-language journal editors stating that their journals' applications to PMC were being rejected. I found thorough evaluations of the scientific and editorial quality of the articles by PMC since November 2014. Scientists and medical librarians review each journal that applies to PMC. Unlike the previous initial stage of the $\mathrm{PMC}$, some journals need to apply twice or three times. Nonetheless, several journals are waiting for re-application, since it usually takes 2 years to re-apply to PMC after rejection. Therefore, editors should meticulously check the scientific quality of their journals.

Third, the feasibility of non-English journals being indexed in PMC: In 2019, PMC announced a new language policy as follows: "PMC will accept applications from non-English MEDLINE journals. For non-MEDLINE journals, NLM requires the primary content to be largely in English before an application can be submitted, as NLM only has the resources to review English-language content for PMC at this time" [5]. Therefore, this provides a good opportunity for editors of non-English journals to apply to PMC. The criterion of the proportion of English articles is known to be half of each issue. For example, in June 2021, six Chinese journals were deposited in PMC (Fig. 2). Many biomedical journals publish

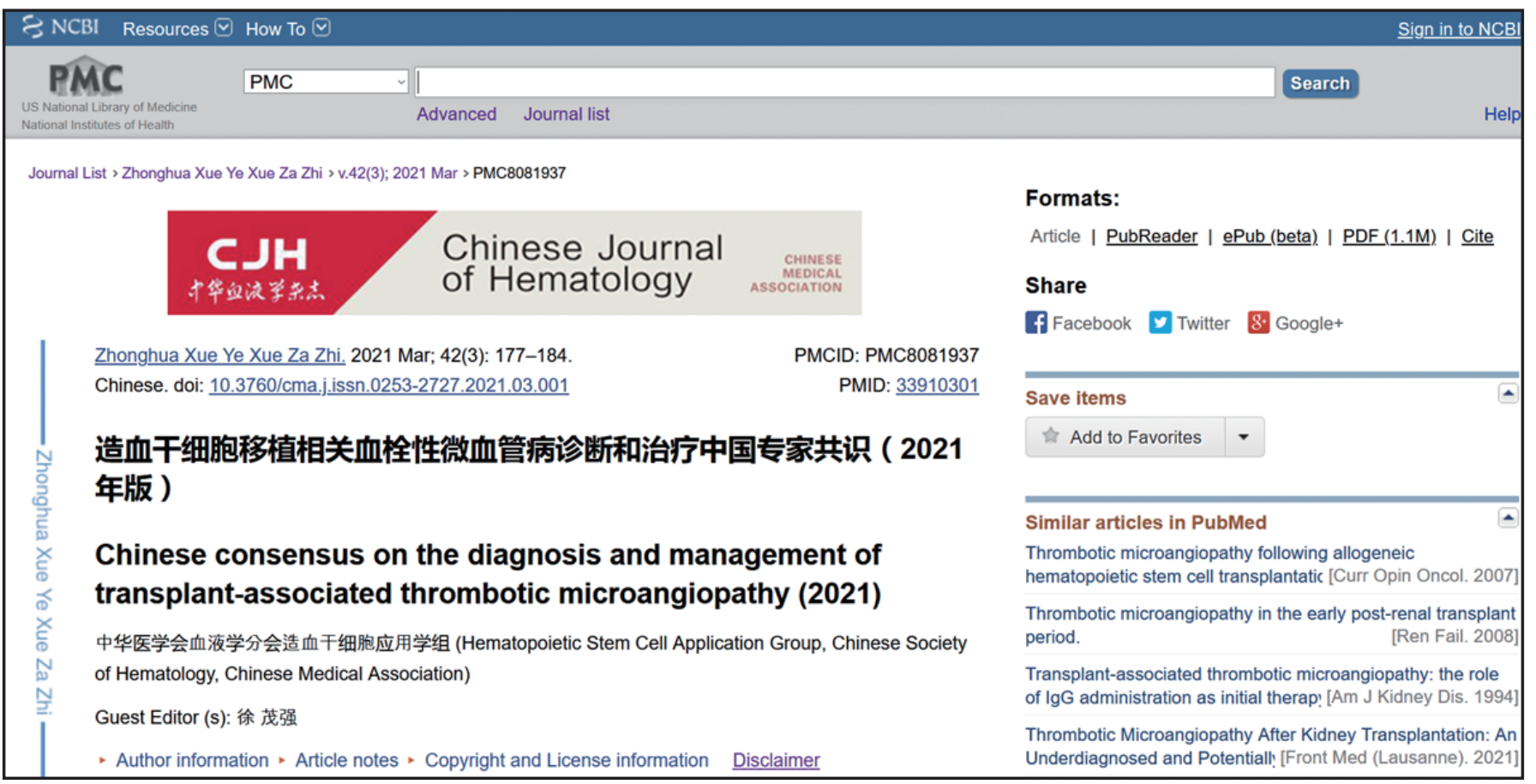

Fig. 2. Zhonghuaxueye xuezazhi=Chinese Journal of Hematology deposited in PubMed Central, available from: https://www.ncbi.nlm.nih.gov/pmc/articles/ PMC8081937/ [cited June 2, 2021]. 


\section{science editing /}

articles written in Korean or English. If half of the articles of each issue are in English, and the number of English-language articles is at least 25, the editor can apply for a journal to be deposited in PMC. The technical quality of PMC XML files from the publishing companies in Korea is excellent, and there are no worries about technical evaluation.

The above three turning points conveyed to editors in Korea the perception that the earlier the conversion to an English-only journal, the easier it is to be indexed in PMC. However, the new non-English language policy announced in 2019 provides an excellent opportunity for the editors of partially Korean-language journals to apply to PMC.

KoreaMed Synapse as benchmarking of PMC

The KAMJE constructed KoreaMed Synapse in 2007 because it was not possible to add Korean-language journals to PMC. KoreaMed Synapse is a platform imitating PMC, the content of which is made up of KAMJE journals. The difficulties faced by local journals include the lack of a platform or literature databases. The aim of KoreaMed Synapse was for articles to be accessed by world researchers through Google or Google Scholar. The early history of Synapse is described as follows: "In August 2007, the Journal of the Korean Ophthalmological Society launched to print DOI prefixes and suffixes on each paper. After that, 17 journals have participated in DOI/Crossref as of October 2007. KAMJE became a sponsoring publisher of DOI/Crossref for KAMJE members. Participation in DOI/ Crossref requires depositing DOI XML to Crossref. Also, the landing page (or response page) should be constructed to hyperlink the full text of reference articles via the DOI system. This series of works require the new landing page database. In November 2007, the database system for landing pages and DOI hyperlinks was completed. This database adopted the PMC XML to show the full text of journals in English or bibliographic data, abstracts, figures, tables, and references of articles from journals in Korean. Therefore, if any journals in Korean move to journals in English, the presentation of fulltext articles will be smoothly transferred to the PMC XML system" (https://synapse.koreamed.org/about/overview.php).

Because many databases' crawling robots crawled KoreaMed Synapse, world researchers' likelihood of accessing KoreaMed Synapse was thought to have increased. Unfortunately, in 2013, the KAMJE decided to exclude journals that did not produce PMC XML by a designated XML producing company. Thereafter, the number of journals steadily decreased until 2020. In April 2020, the new executive board decided to discontinue this monopolistic policy. Therefore, the number of journals that participate in KoreaMed Synapse will dramatically increase starting in July 2021.

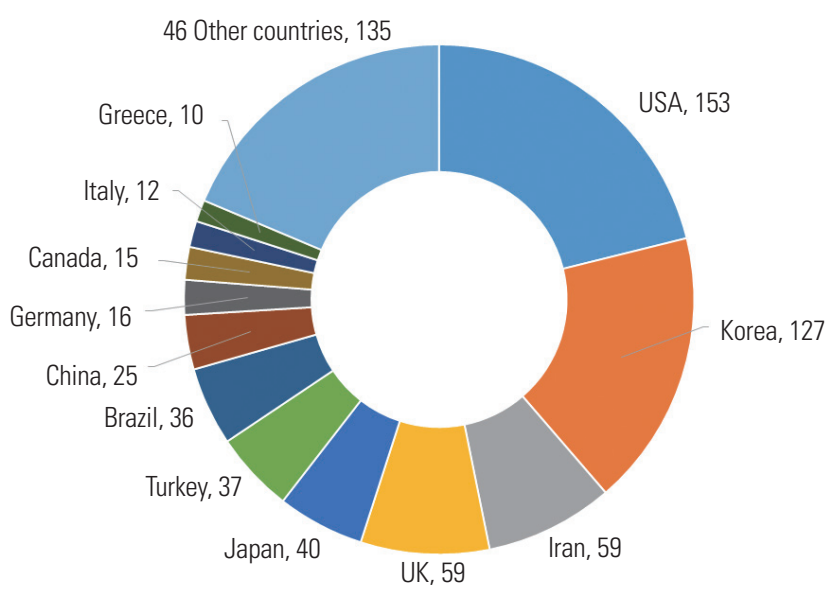

Fig. 3. Distribution of PubMed Central journals without embargo published by academic societies or non-profit organizations according to country in June 2021

The proportion of PMC journals from Korea

Before November 2008, no journal from Korea was indexed in PMC, whereas 29 journals from Korea were deposited in PMC as of June 2010 [4]. Out of 2,199 PMC journals in the category of full participation, immediate free access, no embargo period, no predecessor, and new content, 127 journals (5.8\%) are from Korea as of June 1, 2021 (Dataset 1). That number does not include some journals owned by Korean scholarly societies, but published by commercial publishing companies outside Korea. Scholarly societies or institutes publish 724 (34.2\%) of the 2,119 PMC journals without embargo. If only journals published by scholarly societies or institutes are counted, the proportion of Korean journals increases to $17.5 \%$ out of 724 journals, which is the secondhighest rank according to country. The number of PMC journals without embargo published by scholarly societies or institutes according to country is presented in Fig. 3.

\section{Discussion}

Interpretation: The history of PMC XML file deposition and the launch of KoreaMed Synapse reflect concrete tasks deliberately undertaken by Korean medical editors to upgrade their local society journals to the international level. In particular, the initiation of PMC XML file production in 2006 was a timely step in that process. If I had not begun to produce PMC XML files in 2006, there might have been a delay in the addition of biomedical journals in Korea to PMC, and some journals might have sought out commercial publishing companies for this job.

The fact that Korea is the second highest-ranked country in PMC in terms of journals without embargo published by aca- 
demic societies or institutes reflects a remarkable contribution to PMC itself, as Korean journals have substantially augmented the regional diversity of the PMC database. Many biomedical journals in Korea have applied to PMC, and a substantial number of journals have succeeded in being accepted. Starting in 2021, journals published in Korean and English have been able to apply to PMC, as long as at least half of their articles are in English. The results of applying to PMC are anticipated to be a new milestone for these journals. The history presented in this article can be a model for local society journals worldwide as a way to promote the wide dissemination of biomedical journals' content. In Korea, the involvement of commercial publishing companies in academic publishing has been rare. Therefore, the editor's role is that of an editorpublisher-not merely an editor. This means that editors must both be involved in the review and selection of the manuscripts and play an active role in processes related to publishing. They should understand and implement relevant publishing policies. This is the main difference between their role and that of editors of journals published by commercial companies, where publishing staff work to support the entire publishing process.

The value of PMC for journals in Korea can be summarized as follows. First, PMC has encouraged journal editors to change their language to English-only. Between 2011 and 2019, the number of English-only journals changed from 64 (33.5\%) to $110(57.6 \%)$ among the 191 medical journals registered with the Korean Federation of Science and Technology Societies in 2011 [6].

Second, PMC has helped local journals receive more citations from world researchers, increasing their likelihood of being indexed in international databases, including Scopus and the Web of Science Core Collection. Out of 127 PMC journals, 106 are indexed in Scopus, 54 in SCIE, and 33 in ESCI. All SCIE and ESCI journals are indexed in Scopus except for one. The Journal of Educational Evaluation for Health Professions, which I have edited since 2005, is also indexed in Scopus [7] and ESCI. Only 20 PMC journals are not indexed in any of these three databases, whereas 107 (84.8\%) of the PMC journals in Korea are indexed in at least one international database (Fig. 4). Fifty-three journals (41.7\%) are published as diamond open-access journals with no article processing charge on the author side (Dataset 1 ). The article processing charges of the $127 \mathrm{PMC}$ journals are also listed in $\mathrm{Da}-$ taset 1 .

Third, the number of submissions from researchers from other countries increased, thereby making it possible for journals to achieve international diversity.

Currently, if a new biomedical journal launches, the first goal of the editor is to add the journal to PMC. Editors of Ko-

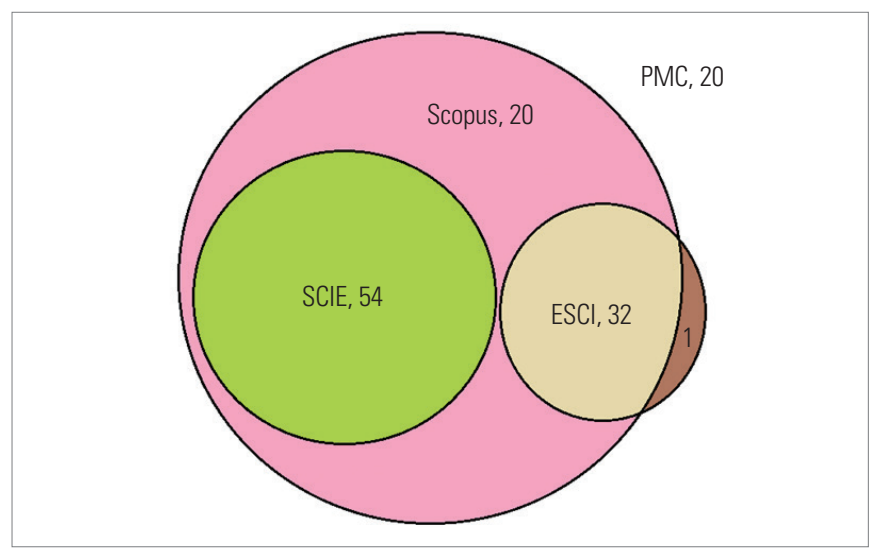

Fig. 4. The proportion of 127 PubMed Central (PMC) journals from Korea without embargo, indexed in the international databases Scopus, SCIE, and ESCl in June 2021.

rean-language journals also began to recruit articles in English to apply to PMC. It is not possible to understand the development of biomedical journals in Korea without considering the role of PMC.

Limitation: The classification of publisher type and country of publication was done by the author's decision; therefore, there may have been unidentified misclassifications.

Conclusion: The implementation of PMC in 2000 influenced local journal editors in Korea who wanted their journals to become top-tier international journals. It provided an excellent opportunity for them to add their journals to PubMed because PMC transferred abstracts to PubMed automatically. Crossref XML deposition in 2007 and full-text XML file deposition to PMC in 2008 were two milestones for biomedical journals in Korea, as these achievements made it possible for the journals to participate in the international scholarly network represented by Crossref and PubMed. Twenty-one years have passed since the launch of PMC, and the number of PMC journals from Korea without embargo reached 127 in June 2021, making Korea the second highest-ranked country in terms of no-embargo PMC journals published by academic societies or institutes.

Since 2019, non-English journals can apply to PMC if at least half of the articles in a single issue are written in English. This provides another excellent opportunity for journal editors in Korea. If a journal is listed in PMC, the non-English full-text articles can be deposited to PMC, and their abstracts are transferred to PubMed. It is time for biomedical editors in Korea to do their best to include their journals in PMC. As the best full-text platform of biomedical journals, PMC is anticipated to be able to sustain its top-tier brand in the future, and the US NLM is expected to continue to support this superb archiving database. Establishing a strategy and plan for 
being listed in PMC is mandatory for biomedical, open-access journal editors not only in Korea, but also all over the world.

\section{Conflict of Interest}

Sun Huh has been the President of the Korean Council of Science Editors since January 17, 2020, but had no role in the decision to publish this article. Except for that, no potential conflict of interest relevant to this article was reported.

\section{Funding}

The author received no financial support for this article.

\section{Data Availability}

Dataset file is available from: the Harvard Dataverse at: https:// doi.org/10.7910/DVN/WODLKV

Dataset 1. PMC journal list downloaded from: https://www.ncbi.nlm.nih.gov/ pmc/about/faq/\#q8 [cited 2021 Jun 1] and analyzed data. a) PMC journal list, b) full participation journal list with only present deposition, c) full participation journal list with only present deposition and with no embargo, d) 724 society or institute journals, e) sorting of the 724 society or institute journals according to country, f) journals from Korea-only list.

\section{Supplementary Material}

Supplementary files are available from the Harvard Dataverse at: https://doi.org/10.7910/DVN/WODLKV

Suppl. 1. KoreaMed technical workshop, 2006 on PMC XML and XSLT Pro- cessor cited from https://www.kamje.or.kr

Suppl. 2. DOI/CrossRef, PMC XML workshop cited from https://www.kamje. or.kr

\section{References}

1. US National Library of Medicine. PubMed Central [Internet]. Bethesda, MD: US National Library of Medicine; 2021 [cited 2021 Jun 1]. Available from: https://www.ncbi. nlm.nih.gov/pmc/

2. Jeong $\mathrm{GH}$, Huh S. Increase in frequency of citation by SCIE journals of non-Medline journals after listing in an open access full-text database. Sci Ed 2014;1:24-6. https:// doi.org/10.6087/kcse.2014.1.24

3. Huh S. To broaden the horizon of the Journal of Educational Evaluation for Health Professions. J Educ Eval Health Prof 2006;3:1. https://doi.org/10.3352/jeehp.2006.3.1

4. Huh S. Medical databases from Korea and abroad. J Korean Med Assoc 2010;53:659-67. https://doi.org/10.5124/jkma.2010. 53.8.659

5. US National Library of Medicine. PMC policies and guidelines [Internet]. Bethesda, MD: US National Library of Medicine; 2021 [cited 2021 Jun 1]. Available from: https://www.ncbi.nlm.nih.gov/pmc/about/guidelines/

6. Seo YJ, Cho HM, Huh S. Changes in bibliographic information associated with Korean scientific journals from 2011 to 2019. Sci Ed 2020;7:11-5. https://doi.org/10.6087/ kcse. 184

7. Huh S. Journal of Educational Evaluation for Health Professions will be accepted for inclusion in Scopus. J Educ Eval Health Prof 2019;16:2. https://doi.org/10.3352/jeehp.2019.16.2 